\title{
Motion-Segmentation-Based Change Detection
}

\author{
Bing Han, William Roberts, Dapeng Wu, Jian Li \\ Department of Electrical and Computer Engineering \\ University of Florida Gainesville, FL 32611 \\ Correspondence author: Prof. Dapeng Wu, \\ wu@ece.ufl.edu, \\ http://www.wu.ece.ufl.edu
}

\begin{abstract}
Detecting regions of change in images of the same scene taken at different times is of widespread interest. Important applications of change detection include video surveillance, remote sensing, medical diagnosis and treatment. Change detection usually involves image registration, which is aimed at removing meaningless changes caused by camera motion. Image registration is a hard problem due to the absence of knowledge about camera motion and objects in the scene. To address this problem, this paper proposes a novel motion-segmentation based approach to change detection, which represents a paradigm shift. Different from the existing methods, our approach does not even need image registration since our method is able to separate global motion (camera motion) from local motion, where local motion corresponds to regions of change while regions with only global motion will be classified as 'no change'. Hence, our approach has the advantage of robustness against camera motion.

Separating global motion from local motion is particularly challenging due to lack of prior knowledge about camera motion and the objects in the scene. To tackle this, we introduce a motion-segmentation approach based on minimization of the coding length. The key idea of our approach is as below. We first estimate the motion field by solving the optical flow equation; then we segment the motion field into regions with different motion, based on the minimum coding length criterion; after motion segmentation, we estimate the global motion and local motion; finally, our algorithm outputs regions of change, which correspond to local motion. Experimental results demonstrate the effectiveness of our scheme.
\end{abstract}

Keywords: Change Detection, Object Tracking, Scene Interpretation, Optical Flow, Minimal Coding Length, Motion Segmentation, Global Motion Estimation.

\section{INTRODUCTION}

Detecting regions of change in images of the same scene taken at different times is of widespread interest due to a large number of applications in diverse disciplines. Important applications of change detection include video surveillance, remote sensing, medical diagnosis and treatment, civil infrastructure, and underwater sensing. Change detection usually consists of three steps, namely, 1) image registration, a.k.a., geometric adjustment, to remove meaningless changes caused by camera motion, 2) radiometric/intensity adjustment to mitigate lighting variation and noise, and 3) stochastic modeling and hypothesis testing, to decide which pixel/area experiences changes of interest. This conventional paradigm involves image registration, which is a challenging problem to solve due to the absence of knowledge about camera motion and objects in the scene. To address this challenge, we propose a novel motion-segmentation based approach to change detection, which represents a paradigm shift. Different from the conventional paradigm, our approach dispenses with image registration since our method is able to separate global motion (camera motion) from local motion, where local motion corresponds to regions of change while regions with only global motion will be classified as 'no change'. Hence, our approach has the advantage of robustness against camera motion.

Separating global motion from local motion is particularly challenging due to lack of prior knowledge about camera motion and the objects in the scene. To tackle this, we introduce a motion-segmentation approach. Motion segmentation [1] is different image segmentation. Image segmentation methods, including edge detection and intensity thresholding, are used to provide information about a single frame [2]. For a sequence of images, a 
complete understanding of the scene can prove very challenging, as the occurrence of multiple moving objects or the appearance of objects at different depths could lead to motion discontinuities. To obtain needed information about the objects' movements or regions of change [3], segmentation can be used to divide a picture/frame of an image sequence into motion-based regions; this process is called motion segmentation.

Motion segmentation is a central constituent of several technologies. The MPEG-4 standard, which is used to compress digital audio and video data [4], describes a content-based manipulation of objects in image sequences. To create an object-based scene representation, different objects in a frame are segmented, typically by way of their motion information. Video querying [5], another new field, aims to automatically classify video sequences based on their content. A common video query task requires retrieving all the images in a database that have similar content to the input image. By providing an indexing scheme that uses the trajectories, shapes, and flow vectors of the moving objects, motion segmentation serves to enhance the performance of video querying. The development of unmanned aerial vehicles [6], a major advancement in reconnaissance, requires scene analysis technology to identify suspicious military vehicles in a video sequence. Objects having different moving velocities or directions can be identified and isolated through segmentation techniques.

\subsection{Literature Review}

Motion segmentation refers to the assignment of groups of pixels to various classes based on the speed and direction of their movements. Most approaches to motion segmentation first seek to compute the optical flow of the image sequence. In [7], Horn and Schunck presented an iterative method to calculate the optical flow. Using the rate of change of the image intensity and by assuming that the brightness function changes smoothly, they attained the flow velocity by minimizing a global error function. Though Horn and Schunck's algorithm achieves a dense optical flow field, their approach remains sensitive to noise. An alternate method is proposed by Lucas and Kanade in [8]. By assuming a locally constant flow, their algorithm achieves improved robustness against noise at the expense of the resolution of their optical field.

Many different methods currently exist to provide segmentation of the flow field. Wang and Adelson described in [9] a method to represent moving objects using sets of overlapping layers. The layers are obtained using a K-means clustering algorithm, and are ordered based on their depth in the image. Furthermore, a velocity map is used to describe the deformation of the layers over time. Several proposed algorithms follow this layerbased approach [10], [11]. In contrast, Schnorr and Cremers [12] presented a variational method, called motion competition, which jointly solves the problems of motion estimation and segmentation for two consecutive frames in a sequence. By their approach, a single energy functional is minimized with respect to the affine motion model in each separate region and to the shape of the separating contour.

All of the above approaches try to solve the motion segmentation problem, but only via motion information. Image intensity information could also be used to find the optimal motion segmentation results. In addition, previous methods fail to provide a robust means by which to extract the global motion from an image sequence.

\subsection{Our Approach}

In this paper, we propose an approach for motion segmentation based on an image's optical flow field. The novelty of our work involves the introduction of minimum coding length as a criterion in clustering or grouping of motion vectors so that a motion field* is segmented into regions of different motion. Furthermore, we propose a heuristic and highly effective method for the estimation of global motion in an image sequence. Based on our techniques for motion segmentation and global motion estimation, we design a change detection algorithm. The key idea of our change detection algorithm is as below. We first estimate the motion field by solving the optical flow equation; then we segment the motion field into regions with different motion, based on the minimum coding length criterion; after motion segmentation, we estimate the global motion and local motion; finally, our algorithm outputs regions of change, which correspond to local motion. Our experimental results demonstrate the effectiveness of our scheme.

The remainder of this paper is organized as follows. In Section 2, we describe a method to estimate the motion field. Section 3 describes our motion segmentation approach, which is based on the minimum-codinglength criterion. In Section 4, we present our scheme for global motion estimation. Section 5 describes our

\footnotetext{
* A motion field is defined as a 2D array, each element of which is a motion vector associated with an image pixel.
} 
motion-segmentation-based change detection algorithm, which is based on the schemes presented in Sections 2 through 4. Section 6 shows the experimental results for the proposed algorithms. Section 7 concludes the paper and points out future directions.

\section{MOTION FIELD ESTIMATION}

Optical flow is a method of estimating the motion of objects within a visual representation. Typically, the motion is represented as a vector indicating the direction and speed of a pixel as it moves across the image. It is a powerful tool for image analysis and interpretation.

The traditional approaches to computing optical flow can be classified into categories: feature-based, correlationbased, and gradient-based. Gradient-based algorithms receive special interest due to their mathematical simplicity and relative computational efficiency. In this paper, we will use a gradient-based algorithm, which was first proposed by Lucas and Kanade [8], to estimate the motion field of an image sequence.

This method seeks to calculate the motion between two image frames which are taken at times $\mathrm{t}$ and $\mathrm{t}+\delta t$ at every pixel position. As a pixel at location $(x, y, t)$ with intensity $I(x, y, t)$ will have moved by $\delta x, \delta y$, and $\delta t$ between the two frames, the following image constraint equation can be given:

$$
I(x, y, t)=I(x+\delta x, y+\delta y, t+\delta t) .
$$

By assuming that movement of objects is small enough, the image constraint at $I(x, y, t)$ can be expanded with a Taylor series:

$$
\begin{gathered}
I(x+\delta x, y+\delta y, t+\delta t)=I(x, y, t) \\
+\frac{\partial I}{\partial x} \delta x+\frac{\partial I}{\partial y} \delta y+\frac{\partial I}{\partial t} \delta t+H . O . T .
\end{gathered}
$$

where H.O.T. stands for the higher ordered terms, which are ignored here. From the above equation, we can achieve

$$
\frac{\partial I}{\partial x} \delta x+\frac{\partial I}{\partial y} \delta y+\frac{\partial I}{\partial t} \delta t=0
$$

which results in

$$
I_{x} V_{x}+I_{y} V_{y}=-I_{t}
$$

where $V_{x}$ and $V_{y}$ are the components of the velocity and $I_{x}, I_{y}$, and $I_{t}$ are the spatial and temporal derivatives of the image at pixel $(x, y, t)$.

This equation is known as the aperture problem of optical flow algorithms. In order to solve this problem, an additional constraint is needed. In Lucas and Kanade's solution, they use a non-iterative method that assumes a locally constant flow. From this, we are able to solve the constrained system of equations, yielding:

$$
A \vec{v}=-b,
$$

where $A$ is the constant flow in a small window of size $m \times m$.

The above algorithm shows that optical flow can be computed by calculating the derivatives of the image in both spatial and temporal dimensions. In order to give more prominence to the center pixel in a window, a Gaussian weighting function is preferably incorporated. This flow model can be further extended to affine image deformations.

For example, Figs. 1 and 2 show two frames from an image sequence, and Fig. 3 shows the calculated optical flow field. To maintain a better visual effect, we only draw the motion vector for each 5 by 5 block. The actual motion field is calculated per pixel.

Unfortunately, the calculated optical flow and the true motion field are in general different, unless restrictions on both the lighting conditions and the objects being imaged are satisfied. As a result, this problem remains largely unsolved. We may further address this issue in our future works. 


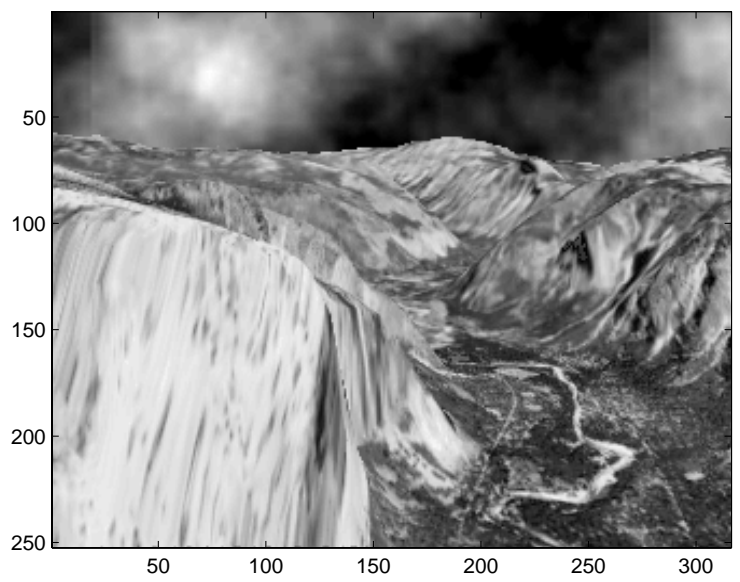

Figure 1. The $2^{\text {nd }}$ frame of the image sequence.

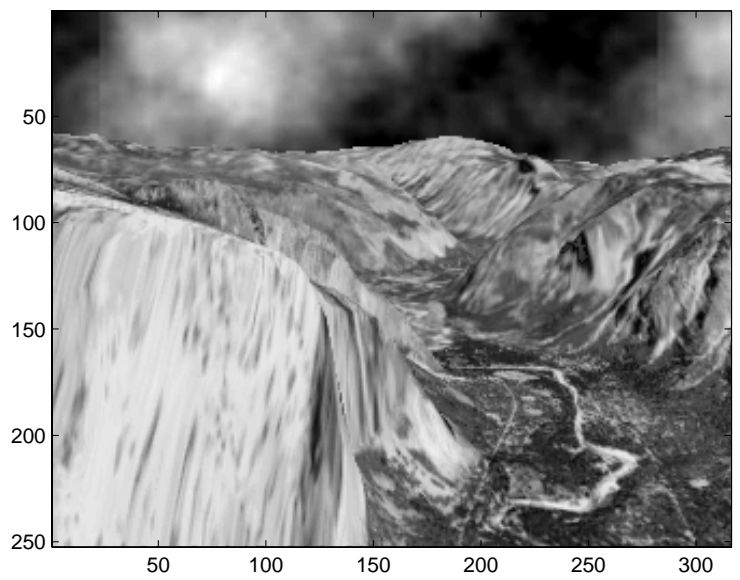

Figure 2. The $4^{\text {th }}$ frame of the image sequence. 


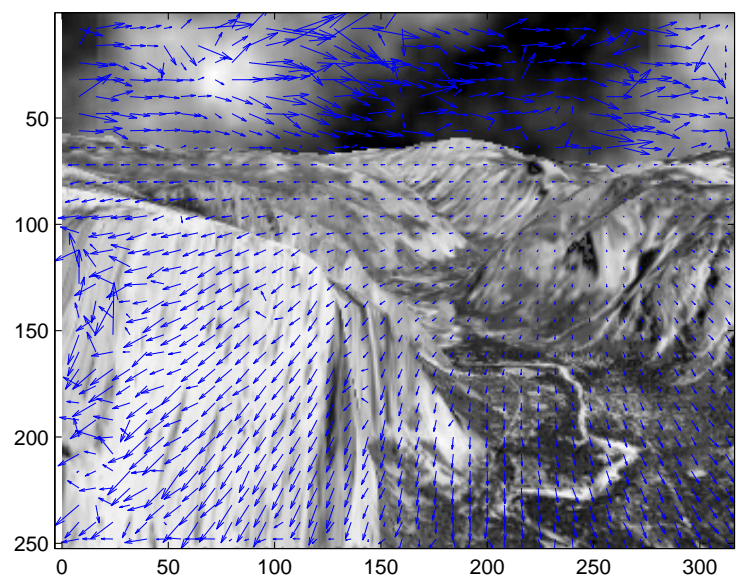

Figure 3. The optical flow field of the selected image sequence.

\section{MOTION FIELD SEGMENTATION}

\subsection{Problem Definition}

The optical flow of a pixel is a 2-dimensional vector indicating how this pixel moves in the scene. The next task for change detection or scene interpretation is to segment the data into homogeneous subsets such that each subset can be more easily represented or interpreted.

A traditional definition of segmentation is to choose a class of models with which to fit each subset. The typical approach to segmenting the data is to simultaneously decompose the mixture of all models into individual ones. Various methods have been proposed to resolve this problem, such as the K-means clustering algorithm and the expectation maximization (EM) algorithm.

In the problem of motion field segmentation, the number of segments is unknown. Therefore, determining the appropriate number of models for the data set becomes a more difficult problem. In order to resolve this issue, we will take a new approach based on minimum description length criterion. Before describing the algorithm, we will first give some further definition of this problem:

- The data $\Lambda \in \mathbb{R}^{M \times N}$ is a set of random samples from a mixture of models.

- The minimum description length criterion is given by

$$
\text { Min.Length }(\Lambda, \theta)=\operatorname{Length}(\Lambda \mid \theta)+\operatorname{Length}(\theta) \text {. }
$$

- The optimal segmentation of the data $\Lambda$ is a partition $\Lambda=\Lambda_{1} \cup \Lambda_{2} \ldots \cup \Lambda_{N}$ such that the overall coding length of the data $\Lambda$ is minimal among all possible segmentations.

In general, the length function Length $(\theta)$ is chosen according to optimal lossless coding of a random source.

\subsection{Coding Length based Segmentation}

In this section, we will show how to calculate the expected coding length after segmenting the data into multiple groups. Suppose we already partitioned the set of data $\Lambda$ into $N$ non-overlapping groups:

$$
\Lambda=\Lambda_{1} \cup \Lambda_{2} \cup \ldots \Lambda_{N} .
$$


From $[13,14]$, we have the expected total number of bits required to encode the data $\Lambda$ according to the above segmentation:

$$
\begin{array}{r}
L^{s}(\Lambda, \Pi)=\sum_{n=1}^{N} L\left(\Lambda_{n}\right)+\left|\Lambda_{n}\right|\left(-\log _{2}\left(\left|\Lambda_{n}\right| / M\right)\right) \\
=\sum_{n=1}^{N} \frac{\operatorname{tr}\left(\Pi_{n}+K\right)}{2} \log _{2} \operatorname{det}\left(I+\frac{K}{\operatorname{tr}\left(\Pi_{n}\right) \varepsilon^{2}} \Lambda \Pi_{n} \Lambda^{T}\right) \\
+\operatorname{tr}\left(\Pi_{n}\right)\left(-\log _{2} \frac{\operatorname{tr}\left(\Pi_{n}\right)}{M}\right),
\end{array}
$$

where the superscript $s$ indicates the coding length after segmentation, and $\Pi_{i}$ denotes the diagonal matrix that encodes the probability of $M$ vectors in $i$ :

$$
\Pi_{i}=\left(\begin{array}{cccc}
\pi_{1 i} & 0 & \cdots & 0 \\
0 & \pi_{2 i} & \ddots & \vdots \\
\vdots & \ddots & \ddots & 0 \\
0 & \cdots & 0 & \pi_{M i}
\end{array}\right) \in \mathbb{R}^{M \times M}
$$

Here, $\pi_{j i}$ denotes the probability that the $j$ th vector belongs to the $i$ th group.

Based on the above assumption, optimal segmentation is the approach that produces the minimal overall coding length $L^{s}(\Lambda, \Pi)$, and can equivalently be viewed as a good approximation to the actual entropy of the segmented data set.

An optimal solution to the coding length equation can be found in a bottom-up manner by merging regions of segments. A detailed proof is given in $[13,14]$.

\subsection{Minimizing the Coding Length}

We next seek to minimize the average coding length, given by

$$
R^{s}(\Lambda, \Pi)=\frac{1}{M} L^{s}(\Lambda, \Pi)
$$

as a function in $\Pi$.

Since the number of groups is unknown, we have to minimize $R^{s}(\Lambda, \Pi)$ over $N \in \mathbb{Z}_{+}$. From previous works, we know that any gradient-based descent algorithm relies on the initialization of data sets in order to converge to a global minimum. Since motion fields do not necessarily satisfy this requirement, it is quite difficult to minimize the rate function directly. Instead, we use a steepest-descent algorithm to minimize the length function $L^{s}(\Lambda, \Pi)$.

This algorithm is presented in Fig. 4. In each step, we choose two subsets of vectors, $\Lambda_{i}, \Lambda_{j} \in \Lambda$, such that by merging these two subsets, the decrement in the coding length is the largest. When the dimension of the space is relatively low, the greedy algorithm usually performs well. However, when the dimension of the subspace becomes high, the greedy algorithm does not always converge to the optimal solution.

\section{GLOBAL MOTION ESTIMATION}

In general, there are two kinds of motion, global motion and local or object motion. Global motion is caused by the movement of the camera and local motion is caused by the movement of objects.

In order to analyze the motion of the object, we have to first identify the global motion. This difficult task is similar to the problem of separating the background from the foreground in a static image. Some researchers have proposed approaches based on motion transformation and image rectification. However, these methods are computationally expensive and fail for motion fields in the presence of inherent noise. In order to solve this problem, we propose a heuristic approach based on the properties of motion segments. 


\section{Algorithm 1 Steepest Descent Minimization $(\Lambda)$}

1. while flag $==$ true do

2. $\quad$ choose distinct sets $\Lambda_{i}, \Lambda_{j} \in \Lambda$ such that $L^{s}\left(\Lambda_{i}, \Lambda_{j}\right)-L^{s}\left(\Lambda_{i} \cup \Lambda_{j}\right)$ is maximum among all possible pairs

3. if $L^{s}\left(\Lambda_{i}, \Lambda_{j}\right)-L^{s}\left(\Lambda_{i} \cup \Lambda_{j}\right)>0$

4. $\quad \Lambda:=\left(\Lambda \backslash\left\{\Lambda_{i}, \Lambda_{j}\right\}\right) \cup\left\{\Lambda_{i} \cup \Lambda_{j}\right\}$

5. else

6. $\quad$ flag $=$ false;

7. end

8. end while

9. return the set $\Lambda$

Figure 4. Steepest Descent Minimization Algorithm

\section{Algorithm 2 Global Motion Estimation $(\Lambda)$}

1. find the motion segment that contains the most number of corners

2. if more than one segment contains corners

3. compare their variance of motion vectors

4. return the segment that has minimal variance

5. else

6. return the only segment

7. calculate the average of all the motion vectors in the segment, which is regarded as the global motion vector

Figure 5. Global Motion Estimation Algorithm

Generally, regions of global motion contain the corners of the scene. Although it is not always the case, however, we still could utilize this assumption combined with the variance statistics of the motion field segments to estimate the global motion. For each output segmentation, we will search for the motion region which contains the most corners of the scene. If there are more than one segments containing corners of the scene, the segment with the minimal variance of motion vectors will be selected to represent the global motion.

Once selected, the global motion is then taken as the average of all the motion vectors within the region. The algorithm is given in Fig. 5.

\section{CHANGE DETECTION}

In this section, we describe our motion-segmentation based change detection algorithm. As shown in Fig. 6, the algorithm takes two images as input; the two images are captured by a camera at different time instants; the camera may experience some motion. Given the two images, in Step 1, the algorithm solves the optical flow equation described in Section 2 and obtains a motion field. In Step 2, the motion field is segmented into regions with different motion by using Algorithm 1 described in Section 3. In Step 3, the global motion vector is estimated by Algorithm 2 described in Section 4. In Step 4, local motion vectors are obtained by removing global motion from the motion obtained in Step 2. Finally, the algorithm outputs 1) a global motion vector, 


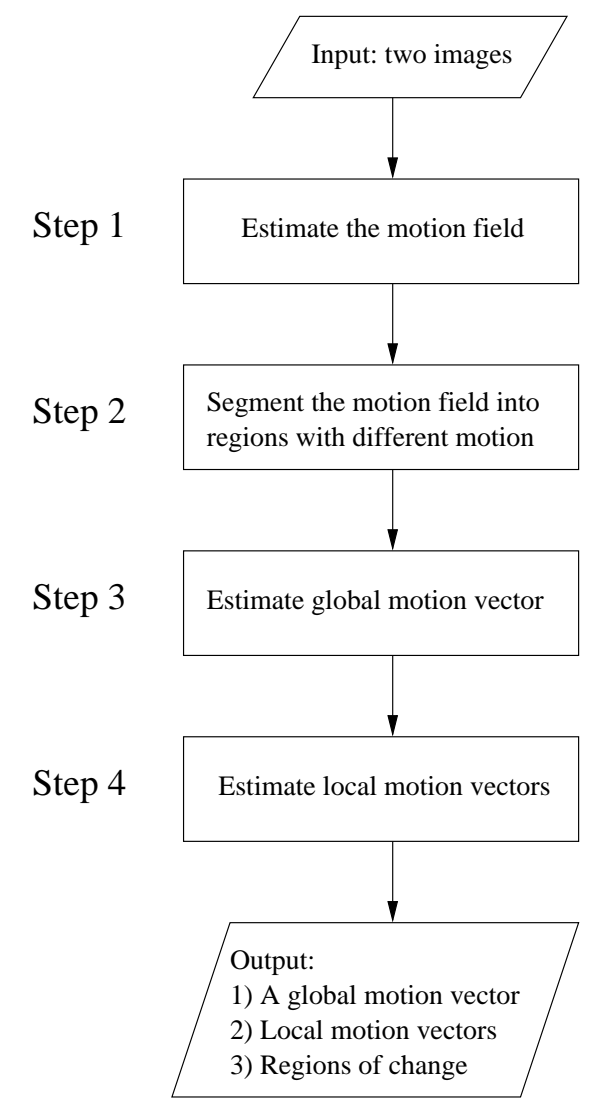

Figure 6. Flow chart of the algorithm for change detection.

2) local motion vectors, and 3) regions of change. Note that regions of change are those that experience local motion.

\section{EXPERIMENTAL RESULTS}

\subsection{Motion Field Segmentation}

To evaluate the proposed algorithm, we applied our segmentation approach to several video sequences containing various types of object motion.

Fig. 7 shows the first frame of an input image sequence containing two boats moving in a translational direction at different speeds along a river. To further complicate the experiment, global motion is introduced to the scene through the camera's movements. In Fig. 8, the optical flow field, computed using the algorithm in [8], is displayed. Finally, Figs. 9 through 12 show the four regions segmented by our proposed algorithm. With only some minor discrepancies between the land and water regions, each object in the sequence is successfully isolated by our approach.

\subsection{Global Motion Estimation}

We now seek to estimate the global motion in the boat sequence by applying our new, heuristic approach. The two segments containing the boats, seen in Figs. 9 and 10, do not contain any corners of the scene, and thus are not considered as candidates. The land and water segments, seen in Figs. 11 and 12, respectively, each contain two corners of the scene. As the variance of motion vectors is smaller in the land region, however, the vectors in this segment will be averaged to obtain an estimate of the global motion. As the land region is the only unmoving region, our method proves effective for this example. 


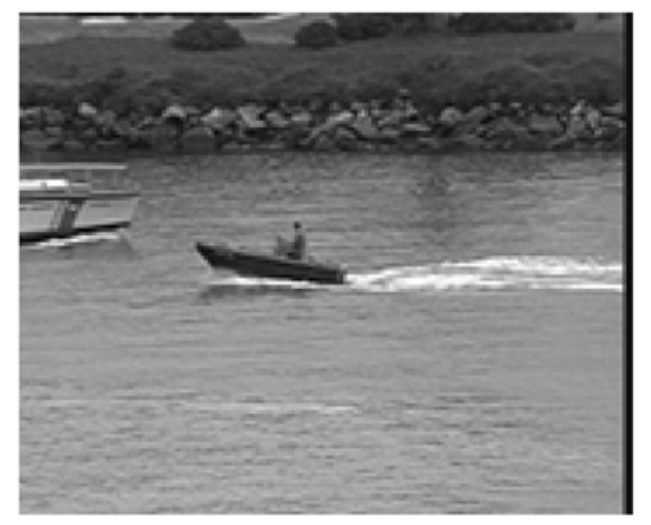

Figure 7 . The $1^{\text {st }}$ frame in the boat image sequence.

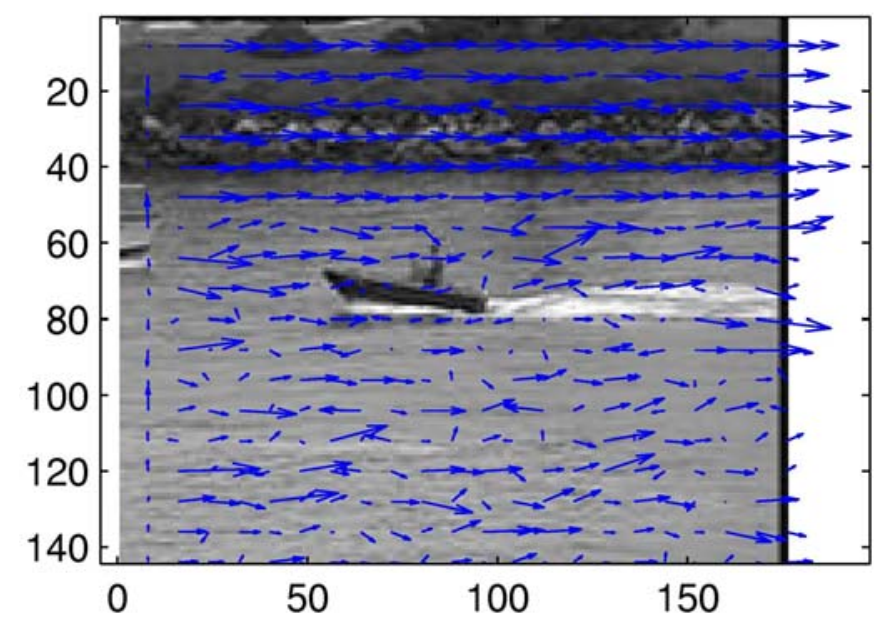

Figure 8. The computed optical flow field of the image sequence. 


\section{CONCLUSION}

In this paper, we have proposed a new algorithm for change detection based on motion segmentation. In our approach, segmentation of motion fields is based on a minimal coding length criterion; initial results have demonstrated superior performance. Furthermore, we introduced a new, heuristic method of extracting the global motion from an image sequence. Based on our techniques for motion segmentation and global motion estimation, we designed a change detection algorithm. Our approach to change detection represents a paradigm shift. Different from the existing methods, our approach does not even need image registration since our method is able to separate global motion (camera motion) from local motion, where local motion corresponds to regions of change while regions with only global motion will be classified as 'no change'. Hence, our approach has the advantage of robustness against camera motion.

Our future directions include improving accuracy of motion segmentation and global motion estimation. As the segmentation algorithm is applied for motion vector fields, the performance of this algorithm is limited by the accuracy of the optical flow field calculated from the input image sequence. In order to improve the performance, texture information could be incorporated into the segmentation approach. Further, we plan to investigate more inherent temporal properties of global motion in order to improve accuracy of global motion estimation.

\section{Acknowledgement}

This work was supported in part by the Air Force Research Laboratory under grant FA8650-06-1-1027.

\section{REFERENCES}

1. P. Bouthemy and E. Francois, "Motion segmentation and qualitative dynamic scene analysis from an image sequence," in International Journal of Computer Vision, pp. 157-182, April 1993.

2. N. R. Pal and S. K. Pal, "A review on image segmentation techniques," in Pattern Recognition, pp. 12771294, 1993.

3. D. W. Murray and B. F. Buxton, "Scene segmentation from visual motion using global optimization," IEEE Trans. Pattern Anal. Mach. Intell. 9(2), pp. 220-228, 1987.

4. D. L. Gall, "Mpeg: a video compression standard for multimedia applications," Commun. ACM 34(4), pp. 46-58, 1991.

5. W. Aref, M. Hammad, A. C. Catlin, I. Ilyas, T. Ghanem, A. Elmagarmid, and M. Marzouk, "Video query processing in the vdbms testbed for video database research," in $M M D B$ '03: Proceedings of the 1st ACM international workshop on Multimedia databases, pp. 25-32, ACM Press, (New York, NY, USA), 2003.

6. R. Pless and D. Jurgens, "Road extraction from motion cues in aerial video," in GIS '04: Proceedings of the 12th annual ACM international workshop on Geographic information systems, pp. 31-38, ACM Press, (New York, NY, USA), 2004.

7. B. K. P. Horn and B. G. Schunck, "Determining optical flow," in Artificial Intelligence,, pp. 185-203, July 1981.

8. B. Lucas and T. Kanade, "Performance of optical flow techniques," in Proc. DARPA IU Workshop, pp. 121130, 1981.

9. J. J. Y. A. Wang and E. H. Adelson, "Representing moving images with layers," in Image Processing, IEEE Transactions on, pp. 625-638, Sep 1994.

10. Y. A. G. D. Borshukov, G. Bozdagi and A. M. Tekalp, "Motion segmentation by multistage affine classification," in Image Processing, IEEE Transactions on, pp. 1591-1594, Nov 1997.

11. N. Jojic and B. Frey, "Learning flexible sprites in video layers," in Proceedings of the IEEE Conference on Computer Vision and Pattern Recognition, pp. I:199-206, (Kauai, Hawaii), 2001.

12. D. Cremers and C. Schnorr, "Motion competition: Variational integration of motion segmentation and shape regularization," in Proceedings of Pattern Recognition, pp. 472-480, (Zurich, Switzerland), Sep 2002.

13. Y. Ma, H. Derkesen, W. Hong, and J. Wright, "Segmentation of multivariate mixed data via lossy coding and compression," to appear in IEEE Transactions on Pattern Analysis and Machine Intelligence, 2007.

14. W. Hong, Hybrid Models for Representation of Imagery Data. PhD thesis, University of Illinois at UrbanaChampaign, Aug. 2006. 


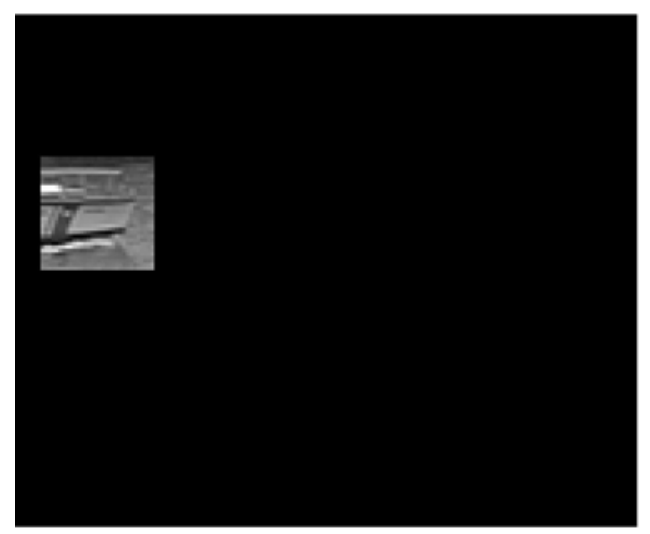

Figure 9. Segmented region from the image sequence containing one of the boats.

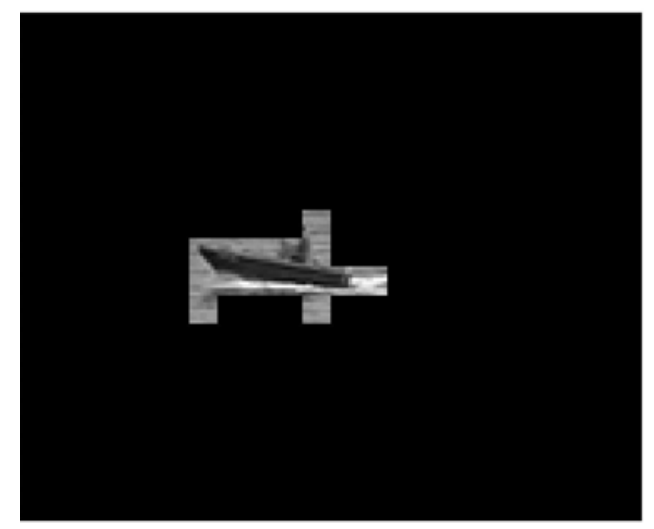

Figure 10. Segmented region from the image sequence containing one of the boats.

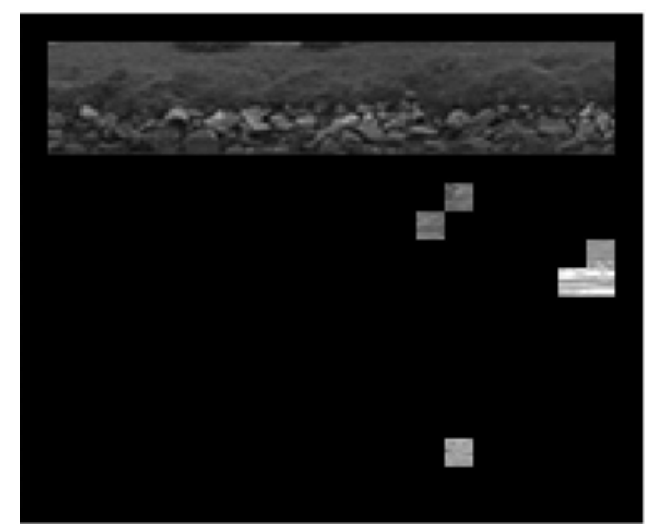

Figure 11. Segmented region from the image sequence containing the land. 


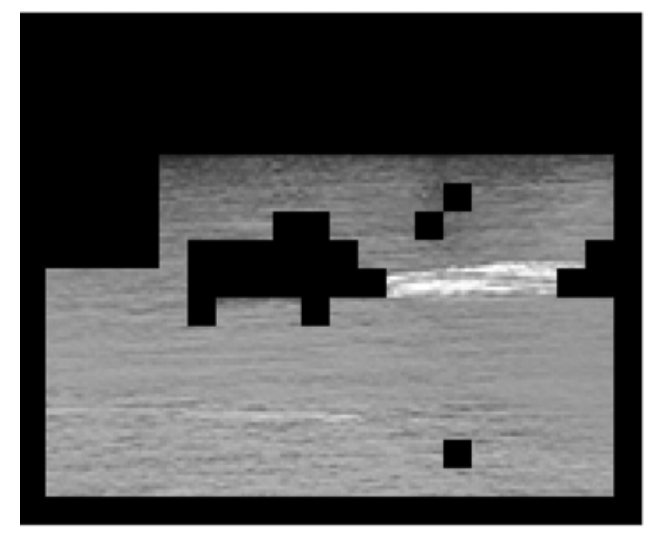

Figure 12. Segmented region from the image sequence containing the water. 